\title{
Hyperspectral phase retrieval: spectral-spatial data processing with sparsity-based complex domain cube filter
}

\author{
Vladimir Katkovnik $\odot$, Igor Shevkunov $\odot,^{*}$ and Karen Egiazarian \\ Tampere University, Faculty of Information Technology and Communication Sciences, \\ Tampere, Finland
}

\begin{abstract}
Hyperspectral (HS) imaging retrieves information from data obtained across broadband spectral channels. Information to retrieve is a $3 \mathrm{D}$ cube, where two coordinates are spatial and the third one is spectral. This cube is complex-valued with varying amplitude and phase. We consider shearography optical setup, in which two phase-shifted broadband copies of the object projections are interfering at a sensor. Registered observations are intensities summarized over spectral channels. For phase reconstruction, the variational setting of the phase retrieval problem is used to derive the iterative algorithm, which includes the original proximity spectral analysis operator and the sparsity modeling of the complex-valued object 3D cube. We resolve the HS phase retrieval problem without random phase coding of wavefronts typical for the most conventional phase retrieval techniques. We show the performance of the algorithm for object phase and thickness imaging in simulation and experimental tests. (C) The Authors. Published by SPIE under a Creative Commons Attribution 4.0 Unported License. Distribution or reproduction of this work in whole or in part requires full attribution of the original publication, including its DOI. [DOI: 10.1117/1.OE.60.1 $.013108]$
\end{abstract}

Keywords: hyperspectral phase retrieval; spectrum analysis; Fourier transform spectrometry; spectral proximity operator.

Paper 20200528 received May 7, 2020; accepted for publication Jan. 11, 2021; published online Jan. 29, 2021.

\section{Introduction}

Hyperspectral (HS) imaging retrieves information from data collected across hundreds to thousands of spectral channels. Conventionally, these data are two-dimensional (2D) images stacked together in 3D cubes, where the first two coordinates are spatial $(x, y)$ and the third one is the spectral channel, which is usually represented by a wavelength $\lambda$.

Over the last few years, HS coherent diffractive imaging (HSCDI) shows a strong progress, for instance, in high-resolution microscopy. Interference between reference and object wavefronts registered as intensity diffraction patterns is used conventionally in spectrally resolved interferometry, ${ }^{1,2}$ Fourier transform holography, ${ }^{3,4}$ and HS digital holography. ${ }^{2,5,6}$ Along with the traditional intensity imaging, HSCDI provides phase imaging, which brings additional information about an object under investigation, e.g., label-free multispectral refractive index estimation. ${ }^{7}$

In this paper, we consider a class of HSCDI in a shearography optical setup, ${ }^{8}$ where two coherent beams, the main (basic) and its time-delay copy, go through a transparent object simultaneously. Thus two identical but phase-shifted broadband patterns of the object are superimposed on the sensor. This scenario, typical for Fourier-transform spectrometry, ${ }^{9}$ leads to the phase loss problem, where a complex-valued 3D object should be reconstructed from indirect intensity observations as solutions of the ill-posed inverse problem. It results in serious amplification of measurement and processing noise. In addition, the high spectral resolution separates the energy obtained by an imaging sensor between many narrow wavebands, which results in small values of signal-to-noise ratio (SNR).

*Address all correspondence to Igor Shevkunov, Igor.Shevkunov@tuni.fi 
HS phase retrieval in the shear geometry has not been studied broadly. We have found only two papers dedicated to such a problem. ${ }^{10,11}$ Kalenkov $^{10}$ realized phase retrieval by special optical system design, in which they produce spatial zero-order filtering of one beam and thanks to it the phase reconstruction comes down to a conventional HS holographic technique of direct phase reconstruction by Fourier transform. Reference 11 is our first solution to the HS phase retrieval problem in the lensless self-reference HS setup. There we perform phase reconstruction by a subsequent forward-backward propagation of HS cube slices by analogy to classical multiwavelength phase retrieval, ${ }^{12}$ with the hypothesis that the object wavefronts produced by neighboring wavelengths are similar. We used this hypothesis for producing phase recalculation from one wavelength to the subsequent one.

In this paper, we propagate HS cube slices all together, without phase recalculation from one slice to another, as it is in Ref. 11. Such processing frees our method from the adjacent slice similarity hypothesis and by that extends the field of operation for objects with a sharp spectral response, where the subsequent slices might be unique.

The contribution of this paper can be summarized as follows. We formalize the HS phase retrieval as a variational optimization problem for noisy Gaussian observations. One of the key components of the derived iterative algorithm is an original proximal operator enabling both the spectral analysis of intensity observations and their denoising. Another important component of the algorithm is the original complex domain filtering of 3D complex-valued object arrays following from the proposed sparsity modeling for complex-valued 3D arrays. It is demonstrated by simulation experiments and processing of experimental data that the HS phase retrieval in the proposed setup can be resolved.

We have published the preliminary results about the proposed algorithm in the conference proceeding. ${ }^{13}$ In this paper, we present more stimulation and as well as experimental results with detailed discussion.

\section{Problem Formulation}

Let $U_{o}(x, y, k) \in \mathbb{C}^{n \times m}$ be a 2D slice of the complex-valued 3D HS cube with spatial coordinates $(x, y)$, a spectral component $k$, and $Q_{K}(x, y)=\left\{U_{0}(x, y, k), k \in K\right\}, Q_{K} \in \mathbb{C}^{n \times m \times l_{K}}$, be the total spectral cube composed of $l_{K}$ spectral 2D slices. The size of the cube is $n \times m \times l_{K}$.

The lines of $Q_{K}(x, y)$ contain $l_{K}$ spectral observations corresponding to the coordinate $(x, y)$. This is a HS model of the object $U_{0}$.

The squared magnitude (intensity) of observations may be written as

$$
Y_{t}=\sum_{k \in K}\left|U_{t, k}\right|^{2}, \quad U_{t, k}=A_{t, k} U_{o, k}, \quad t \in T .
$$

Here we use vector representations for the slices $U_{0}(x, y, k), U_{o, k} \in \mathbb{C}^{N}, N=n m$, and $A_{t, k} \in$ $\mathbb{C}^{M \times N}$ are linear operators of image formation modeling the propagation of $2 \mathrm{D}$ object wavefronts from the object plane to the sensor plane.

Thus $U_{t, k}=A_{t, k} U_{o, k} \in \mathbb{C}^{M}$ are the complex-valued signals, which intensities are registered by the sensor as $Y_{t} \in \mathbb{R}_{+}^{M}$. The summation on $k$ in Eq. (1) says that the measurements are intensities calculated over the entire spectral components. The squared absolute value $|\cdot|^{2}$ in Eq. (1) is element-wise for components of the vectors $U_{t, k}$. For the additive noise case, the intensity observations become

$$
Z_{t}=Y_{t}+\varepsilon_{t}, \quad t \in T,
$$

where $\varepsilon_{t}$ stays for a Gaussian noise $\sim \mathcal{N}(0, \sigma)$.

The HS phase retrieval is formulated as a reconstruction of the HS object cube $Q_{K}(x, y)$ from the intensity observations $Y_{t}$ or $Z_{t}, t \in T$.

A dependence of the object $U_{o}(x, y, k)$ on the spectral $k$ is a specific feature of the considered phase retrieval setup as compared with the phase retrieval for a monochromatic object $U_{o}$ with observations $Z_{t}=\left|A_{t} U_{o}\right|^{2}+\varepsilon_{t}$, or with the recent multispectral phase retrieval ${ }^{14}$ from 
observations $Y_{t}=\sum_{k \in K}\left|A_{t, k} U_{o}\right|^{2}$, where the object $U_{o}$ is again invariant in the spectral domain and only the propagation operator is varying on $k$.

In this paper, we restrict a class of operators $A_{t, k}$ to the form appeared in Fourier transform spectrometry ${ }^{15}$ with the intensities $Y_{t}$ in the form:

$$
\begin{aligned}
& Y_{t}=\sum_{k \in K}\left|\left(1+e^{-j 2 \pi k t}\right) A_{k} U_{o, k}\right|^{2}, \\
& A_{t, k}=\left(1+e^{-j 2 \pi k t}\right) A_{k}, \quad t \in T .
\end{aligned}
$$

Due to this $A_{t, k}$, two identical but phase-shifted copies of the object wavefront $U_{o, k}$ are superimposed on the sensor plane: main $A_{k} U_{o, k}$ and phase-shifted $e^{-j 2 \pi k t} A_{k} U_{o, k}$.

\section{Algorithm Development}

\subsection{Approach}

We assume the following for sampling on $t$ and $k$ in the above observation model, $K=0,1, \ldots, N / 2-1, T=0,1, \ldots, N-1$, then

$$
Y_{t}=2 \sum_{k \in K}\left[1+\cos \left(\frac{2 \pi}{N} k t\right)\right]\left|B_{k}\right|^{2}, \quad t \in T,
$$

where $B_{k}=A_{k} U_{o, k}, N$ is a number of observations and the integer discrete frequency $k$ covers the low-frequency interval $\{0,1, \ldots, N / 2-1\}$.

The restriction of this frequency interval to length $N / 2$ follows from the periodicity on $t$ of the observation function Eq. (5). Provided that $\left|B_{k}\right|^{2}=0$ for $k=N / 2, \ldots, N-1$, the observations $\left\{Y_{t}\right\}$ uniquely define the intensity spectrum $\left|B_{k}\right|^{2} \in K$ (see Ref. 16).

Let us replace the noisy observations $Z_{t}$ by the differences $\Delta Z_{t}=Z_{t}-\frac{1}{N} \sum_{t=0}^{N-1} Z_{t}$. For large $N$, the noise level in the mean value $\frac{1}{N} \sum_{t=0}^{N-1} Z_{t}$ is of the order smaller than that in $Z_{t}$, and we can assume that

$$
\Delta Z_{t}=Z_{t}-\frac{1}{N} \sum_{t=0}^{N-1} Y_{t}=2 \sum_{k=1}^{N / 2-1} \cos \left(\frac{2 \pi}{N} k t\right)\left|B_{k}\right|^{2}+\varepsilon_{t},
$$

since $\frac{1}{N} \sum_{t=0}^{N-1} \cos \left(\frac{2 \pi}{N} k t\right)=\delta_{k, 0}$, where $\delta_{k, 0}$ is the Kronecker symbol.

Then the criterion proposed for the algorithm design can be given in the form:

$$
\begin{aligned}
J= & \frac{1}{\sigma^{2}} \sum_{t=0}^{N-1}\left\|\Delta Z_{t}-2 \sum_{k=1}^{N / 2-1}\left|B_{k}\right|^{2} \cos \left(\frac{2 \pi}{N} k t\right)\right\|_{2}^{2}+\frac{1}{\gamma} \sum_{k=1}^{N / 2-1}\left\|B_{k}-A_{k} U_{o, k}\right\|_{2}^{2} \\
& +f_{\text {reg }}\left(\left\{U_{o, k}\right\}_{1}^{N / 2-1}\right) .
\end{aligned}
$$

The first summand stays for the minus log-likelihood provided a Gaussian noise. The second summand $J$ in the criterion is penalizing the difference between $B_{k}$ and $A_{k} U_{o, k}$ with the weight $1 / \gamma, \gamma>0$. The last third item is a penalty using the sparsity hypothesis on the cube of the object images $\left\{U_{o, k}\right\}_{1}^{N / 2-1}$. The object reconstruction is obtained by minimizing $J$ on $\left\{U_{o, k}\right\}_{1}^{N / 2-1}$. The wavefronts at the sensor plane $B_{k}$ serve as splitting variables in this optimization, separating the observations from the object images.

Note that the zero-order DC term of the object $U_{o, 0}$ is dropped from the consideration of Eq. (7) as we are interested only in higher-order components of the spectrum.

The developed algorithm iterates $\min _{\left\{B_{k}\right\}} J$ on $B_{k}$ provided given $\left\{U_{o, k}\right\}_{1}^{N / 2-1}$ and $\min _{\left\{U_{o, k}\right\}} J$ provided given $\left\{B_{k}\right\}$. Minimization on $B_{k}$ concerns the two first summands in Eq. (7). We show that this minimization is implemented by the derived proximity operator explicitly separating the 
spectral wavefronts $B_{k}$ at the sensor plane. Minimization on $U_{o, k}$ concerns the last two summands in Eq. (7). In this paper, we prefer to do not to formalize the penalty function $f_{\text {reg }}\left(\left\{U_{o, k}\right\}_{1}^{N / 2-1}\right)$ but to use the special high-quality complex domain filter. This approach is in line with the recent tendency in inverse imaging to use high-quality filters instead of regularizers formally obtained by solving optimization problems.

\subsection{Minimization on $B_{1}$}

For minimization $\min _{\left\{B_{l}\right\}} J$, we solve the equations $\partial J / \partial B_{l}^{*}=0, l=1, \ldots, N / 2-1$, what leads to the set of the complex-valued equations for $B_{l}$ :

$$
\left[\frac{-4}{\sigma^{2}} \sum_{t=0}^{N-1} \Delta Z_{t} \cos \left(\frac{2 \pi}{N} l t\right)+\frac{4 N}{\sigma^{2}}\left|B_{l}\right|^{2}+\frac{1}{\gamma}\right] B_{l}=\frac{1}{\gamma} A_{l} U_{o, l}
$$

Derivation of Eq. (8) can be seen in Appendix A. Inserting $B_{l}=\left|B_{l}\right| e^{j \varphi_{B_{l}}}$ in Eq. (8), we may conclude that

$$
\varphi_{B_{l}}=\varphi_{A_{l} U_{o, l}},
$$

where $\varphi_{A_{l} U_{o, l}}$ is the phase of $A_{l} U_{o, l}$.

This result follows from Eq. (8) because the expression in square brackets is real valued. Further, the magnitude $\left|B_{l}\right|$ is defined as a non-negative solution of the polynomial equation, cubic with respect to $\left|B_{l}\right|$ :

$$
\left[\frac{-4}{\sigma^{2}} \sum_{t=0}^{N-1} \Delta Z_{t} \cos \left(\frac{2 \pi}{N} l t\right)+\frac{4 N}{\sigma^{2}}\left|B_{l}\right|^{2}+\frac{1}{\gamma}\right]\left|B_{l}\right|-\frac{1}{\gamma}\left|A_{l} U_{o, l}\right|=0 .
$$

There is no second power in this equation and the free term is negative, then there is only one positive solution $\left|B_{l}\right|,{ }^{17}$ which is computed by Cardano's formula.

The calculations in Eqs. (8)-(10) are produced in the pixel-wise manner for each pixel separately: the amplitudes for $B_{l}$ are given by Eq. (10) and the phases by Eq. (9).

This solution of $\min _{\left\{B_{l}\right\}} J$ can be interpreted as the proximity operator ${ }^{14,18}$ with a compact notation:

$$
B_{l}=\operatorname{prox}_{f \gamma}\left(A_{l} U_{o, l}\right), \quad l=1, \ldots, N / 2-1,
$$

where $\gamma>0$ is a parameter of the quadratic item in Eq. (7) and $f$ stays for the minus loglikelihood part of the criterion Eq. (7), $f \triangleq \frac{1}{\sigma^{2}} \sum_{t=0}^{N-1}\left\|\Delta Z_{t}-2 \sum_{k=1}^{N / 2-1}\left|B_{k}\right|^{2} \cos \left(\frac{2 \pi}{N} k t\right)\right\|_{2}^{2}$.

The proximity solution $\left\{B_{l}\right\}$ resolves two problems. First, complex domain spectral components $B_{l}$ are extracted from the intensity observations. Thus we yield the spectral analysis of the observed total intensities. Second, the noisy observations are filtered with the power controlled by the parameter $\gamma$ compromising the noisy observations $Z_{t}$ and the power of the predicted signal $A_{l} U_{o, l}$ at the sensor plane.

The calculation of the cosine transform in Eqs. (8) and (10) can be produced using FFT (Proposition 2 in Ref. 16). In MATLAB notation, it looks as follows:

$$
\frac{1}{N} \sum_{t=0}^{N-1} \Delta Z_{t} \cos \left(\frac{2 \pi}{N} k t\right)=\operatorname{real}\left[\operatorname{ifft} 2\left(\Delta Z_{t \in T}\right)\right], \quad \text { for } k=1,2, \ldots, N / 2-1
$$

where $\Delta Z_{t \in T}$ is a sequence $\Delta Z_{t}, t=0, \ldots, N-1$ and ifft2 stays for the 2D inverse FFT.

This equation is valid for the nonsymmetric sampling interval $t \in T=\{0,1, \ldots, N-1\}$. For the symmetric $T=\{-N / 2,-N / 2+1, \ldots, N / 2-1\}$, the formula with FFT is different (Proposition 4 in Ref. 16): 


$$
\frac{1}{N} \sum_{t=-N / 2}^{N / 2-1} \Delta Z_{t} \cos \left(\frac{2 \pi}{N} k t\right)=\operatorname{real}\left\{\operatorname{ifft} 2\left[\operatorname{fftshift}\left(\Delta Z_{t \in T}\right)\right]\right\}, \quad \text { for } k=1,2, \ldots, N / 2-1
$$

where "fftshift" denotes the MATLAB shift operator in frequency domain. This symmetric sampling is often used in applications. In the noiseless scenario, Eqs. (12) and (13) give precise values of $\left|B_{k}\right|^{2}$, the intensity spectrum of observations.

\subsection{Regularization by Sparsity-Based Filters}

Let $U_{o}(x, y, k)$ be a transfer function of a transparent thin object. The phase of this object can be written in the form: ${ }^{19}$

$$
\varphi_{U_{o}}(x, y, \lambda)=\frac{2 \pi}{\lambda} h(x, y)\left(n_{\lambda}-1\right)
$$

where $h(x, y)$ is the object thickness invariant on wavelength $\lambda$, and the phase of $\varphi_{U_{o}}$ is smooth with respect to wavelength $\lambda$ in the denominator of this equation for a visual interval of wavelengths. The refractive index $n_{\lambda}$ is a slowly varying function of wavelength for many materials. Thus the phase of $\varphi_{U_{o}}$ as defined by Eq. (14) is a slowly varying function of wavelength and the object slices $U_{o}(x, y, k)=b_{U_{o}}(x, y) \exp \left(j \varphi_{U_{o}}\right)$ close to each other for nearby wavelengths. Here $b_{U_{o}}$ is an amplitude of the slice.

This simple example, being of a general nature, allows to assume that on many occasions the object slices $U_{o}(x, y, k)$ are slowly varying functions of wavelength, which means that these slices are similar for nearby $k$. Then the spectral lines of $Q_{k}(x, y)$ live in $l_{k}$-dimensional subspaces with $l_{k} \ll l_{K}$. Therefore, the concept of sparsity in the spectral domain can be applied for the modeling of this phenomenon.

In this paper, we exploit the spectral sparsity hypothesis in the special filter designed for joint filtering of spectral slices of the complex-valued object cube. This filter is used as a regularizer of the inverse problem instead of formalizing the last regularization item in the criterion $J$.

This approach is in-line with the recent progress in computational imaging where efficient plug-and-play filters have been recognized as powerful tools for prior and regularization to resolve inverse imaging problems. ${ }^{20,21}$

The following algorithm is exploited for the joint sparsity-based processing of $3 \mathrm{D}$ HS cubes:

$$
\left\{\hat{U}_{o, k}, k \in K\right\}=\operatorname{CCF}\left\{U_{o, k}, k \in K\right\} \text {. }
$$

Complex domain cube filter (CCF) processes 3D cube data $\left\{U_{o, k}, k \in K\right\}$ jointly and provides $3 \mathrm{D}$ estimates $\left\{\hat{U}_{o, k}, k \in K\right\}$ for all $k \in K$. The CCF algorithm starts from the singular value decomposition (SVD) analysis of the HS cube. As a next step, preliminary estimates of complex-valued signal and noise correlation matrices are used to select a small-sized subset (eigenimages) of the SVD eigenvectors that best approximate the signal subspace in the least square error sense. Further, 2D complex domain filtering is applied to the denoising of this small number of eigenimages. The complex domain block-matching $3 \mathrm{D}$ algorithm ${ }^{22,23}$ is used for this filtering of eigenimages. Finally, the filtered eigenimages are used to reconstruct the estimates for the entire 3D cube, i.e., for $k \in K$. Overall, CCF can be classified as the adaptive SVD algorithm with optimal selection of a small number of eigenimages.

This CCF algorithm is a complex domain modification of the fast algorithms developed for real-valued HS observations in Refs. 24 and 25. The modification of this algorithm for complex domain HS data is produced recently in Refs. 26 and 27. A sliding window version of CCF was developed for objects with discontinuous and fast varying spectral characteristics. ${ }^{27}$

\subsection{HS Phase Retrieval Algorithm}

Table 1 presents a block scheme of the developed algorithm. The calculation of the initial spectral guess $U_{o, k}^{(0)}$ uses the complex-valued $2 \mathrm{D}$ object model with the wavelength-varying phase 
Table 1 HS phase retrieval algorithm.

\begin{tabular}{|c|c|}
\hline & Initialization: $U_{o, k}^{(0)}, k \in K$ \\
\hline & Main iterations: For $s=1,2, \ldots$, max $_{\text {iter }}$ do \\
\hline \multirow[t]{2}{*}{1.} & Forward propagation: \\
\hline & $U_{t, k}^{(s)}=A_{k} U_{o, k}^{(s)}, k \in K$ \\
\hline \multirow[t]{2}{*}{2.} & Proximity operation: \\
\hline & $B_{k}^{(s)}=\operatorname{prox}_{f_{\gamma}}\left[U_{t, k}^{(s)}\right], k \in K$ \\
\hline \multirow[t]{2}{*}{3.} & Backward propagation: \\
\hline & $U_{o, k}^{(s)}=A_{k}^{\#} B_{k}^{(s)}, k \in K$ \\
\hline \multirow[t]{4}{*}{4.} & Spectral filtering by CCF: \\
\hline & $\left\{U_{o, k}^{(s+1)}, k \in K\right\}=\operatorname{CCF}\left\{U_{o, k}^{(s)}, k \in K\right\}$ \\
\hline & Output: \\
\hline & $U_{o, k}^{\left(\max _{\text {iter }}+1\right)}, k \in K$. \\
\hline
\end{tabular}

according to Eq. (14) obtained from an uniform initial guess for $h(x, y)$. Stage 1 is the forward propagation from the object plane to the sensor plane according to the operator $A_{k}$. In stage 2 , the proximity operator produces the spectral analysis of the intensity observations and provides the updated version of the wavefront at the sensor plane defined as $B_{k}^{(s)}$. In stage 3, the backward propagation from the sensor plane to the object plane gives the update for the object spectral image $U_{o, k}^{(s)}$. In stage 4, the CCF algorithm produces the joint filtering of the object spectral estimates using optimal SVD eigenimages and updates the object cube estimate as $U_{o, k}^{(s+1)}$. These iterations are repeated $\max _{\text {iter }}$ times.

The backward propagation operator $A_{k}^{\#}$ at stage 3 is an inverse operator for the forward propagation $A_{k}$.

The observation noise is filtered at stage 2 by the proximity operator, whereas the noisy components in the object reconstructions are filtered by CCF.

\subsection{On Interpretation of Hyperspectral Complex Domain Data}

Intensity HS imaging in remote sensing technology mainly works with reflected signals and enables a lot of applications requiring fine identification of materials and physical parameters. HS complex domain sensing is of different nature and of different interpretations. Let us discuss this issue assuming that the specimen of interest is thin and transparent. The proposed and discussed algorithm estimates both the amplitude and phase of the specimen and both estimates are spectral. The amplitude gives information on specimen transparency and material density. This data can be interpreted in a manner similar to that used for HS intensity data.

The reconstructed (measured, imaged) phase, in general, differs essentially from Eq. (14) as this object phase is replaced by the corresponding wrapped phase $\operatorname{wrap}\left[\varphi_{U_{o}}(x, y, \lambda)\right]$, where $\operatorname{wrap}(\varphi)=\bmod (\varphi+p i, 2 p i)-p i$ is the operator wrapping the phase $\varphi$ to the interval $[-\pi, \pi)$.

In what follows, we assume that the phase $\varphi_{U_{o}}(x, y, \lambda)$ belongs to the basic phase interval $[-\pi, \pi)$, then the phase measurements are indeed equal to the object phase. This assumption simplifies the discussion. The reconstructed HS phase $\varphi_{U_{o}}(x, y, \lambda)$ according to Eq. (14) defines a product of two specimen parameters: thickness $h$ and refractive index $n_{\lambda}$ :

$$
\varphi_{U_{o}}(x, y, \lambda) \frac{\lambda}{2 \pi}=h(x, y)\left(n_{\lambda}-1\right) .
$$


Calculating in Eq. (16) the averages of spatial variables $(x, y)$ and spectral $\lambda$, we may separate estimates of thickness and refractive index in the following way:

$$
\begin{aligned}
\operatorname{mean}_{\lambda}\left[\varphi_{U_{o}}(x, y, \lambda) \frac{\lambda}{2 \pi}\right] & =h(x, y) c_{n}, \\
\operatorname{mean}_{(x, y)}\left[\varphi_{U_{o}}(x, y, \lambda) \frac{\lambda}{2 \pi}\right] & =n_{\lambda} c_{h},
\end{aligned}
$$

where $c_{n}=\operatorname{mean}_{\lambda}\left(n_{\lambda}-1\right)$ and $c_{h}=\operatorname{mean}_{h}[h(x, y)]$ are the constants.

These equations give estimates for variations of $h(x, y)$ and $n_{\lambda}$ within invariant factors $c_{n}, c_{h}$. These reconstructions can be useful for many applications, e.g., for phase plate calibration to find variations in a surface and in the optical properties of materials.

If $h(x, y)$ or $n_{\lambda}$ are given, the estimates for $h(x, y)$ and $n_{\lambda}$, respectively, take the form:

$$
\begin{aligned}
& h(x, y)=\frac{1}{2 \pi} \operatorname{mean}_{\lambda}\left[\varphi_{U_{o}}(x, y, \lambda) \lambda /\left(n_{\lambda}-1\right)\right], \\
& n_{\lambda}-1=\frac{1}{2 \pi} \operatorname{mean}_{(x, y)}\left[\varphi_{U_{o}}(x, y, \lambda) \lambda / h(x, y)\right] .
\end{aligned}
$$

A much more complex case occurs when we need to measure the refractive index varying spatially. Then $\varphi_{U_{o}}(x, y, \lambda)=\frac{2 \pi}{\lambda} h(x, y)\left[n_{\lambda}(x, y)-1\right]$, and for estimation of refractive index $n_{\lambda}(x, y)$ we need measurements or extra information on object thickness.

Provided a fixed specimen thickness, we have a refractive index estimate in the explicit form $n_{\lambda}(x, y)=1+\frac{1}{2 \pi} \varphi_{U_{o}}(x, y, \lambda) \lambda / h(x, y)$.

If the thickness is slowly varying on $(x, y)$, then the windowed on spatial coordinates HS phase estimates can be used to image fast variations in $n_{\lambda}(x, y)$.

All estimates discussed in this section are implemented as a postprocessing of HS cube phase $\varphi_{U_{o}}(x, y, \lambda)$ obtained by the proposed algorithm.

If the variations of the object's phase go beyond the basic interval $[-\pi, \pi)$, the wrapping effect becomes essential and the above equations for the thickness and refractive index do not work. The phase unwrapping ${ }^{28}$ is one instrument to resolve the problem, otherwise, a proper interpretation of phase imaging, even visual, without attempts to separate thickness and refractive index, may become problematic. As far as we may conclude from publications, modern phase imaging, at least in biomedicine, is restricted to phase imaging in the basic interval $[-\pi, \pi) .{ }^{29}$

\section{Results}

Simulation and experimental studies of the algorithm performance have been produced for various system parameters and various objects to be reconstructed. Some of these results are shown and discussed in what follows.

\subsection{Simulation Tests}

We model a transparent phase specimen assuming an invariant amplitude and phase defined by Eq. (14), where $h(x, y)$ is varying according to the USAF test image [see Figs. 1(a) and 1(b)]. The refractive index $n_{\lambda}$ is calculated for each $\lambda$ according to Cauchy's equation ${ }^{30}$ with coefficients taken for the glass BK7. ${ }^{31}$ As an illumination source, we model a broadband light beam in the range of $\Lambda=[450: 900] \mathrm{nm}$ with a uniform intensity distribution. The number of wavelengths is 250 . The light beam goes through the specimen and after freely propagates to the sensor where the intensity observations are registered as a 3D cube $Y_{t}(x, y)$ of length $N=2000$, which corresponds to $N$ steps of the phase-shift modulation Eq. (5). We implement this modulation according to the simulated motion of the phase delay stage of the Fourier spectrometer. Each step of this simulated motion was equal to $100 \mathrm{~nm}$ to resolve the smallest wavelengths of the spectral range of the light beam. ${ }^{15}$ The wavelength-dependent image formation 


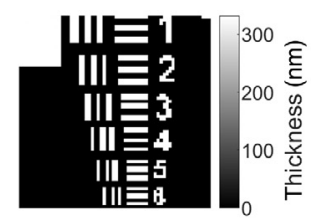

(a)

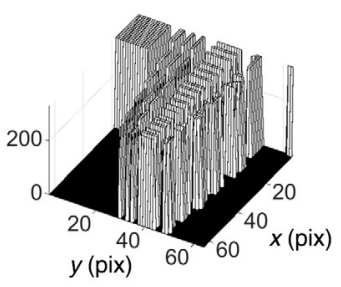

(b)

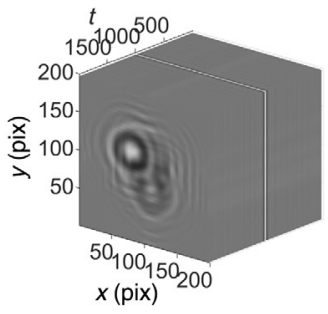

(c)

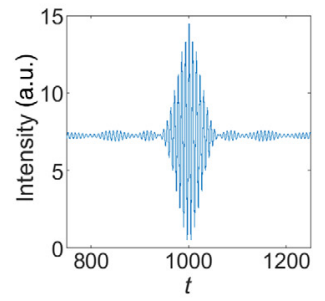

(d)

Fig. 1 The true thickness of the transparent phase object: (a) 2D and (b) 3D images; (c) 3D noisy intensity observations: diffractive data cube $Z_{t}, t \in T$; and (d) the intensity distribution for the central pixel of the $3 \mathrm{D}$ data cube $Z_{t}$ as a function of the experiment number $t$ (slice number in the data cube).

operator $A_{k}$ is calculated according to the angular spectrum propagation technique. ${ }^{19}$ A distance from the specimen to the sensor was equal to $10 \mathrm{~mm}$ and the camera pixel size was $3.45 \mu \mathrm{m}$.

The proximity HS analysis is produced for 250 wavelengths. However, we use only 50 of these wavelengths (range [680:820] nm) corresponding to the forthcoming physical experiments, where only spectra of higher signal-to-noise ratio are exploited for phase retrieval processing. Noisy observations are created according to Eq. (2).

The registered noisy observation cube $Z_{t}(x, y)$ is demonstrated in Fig. 1(c) and a single-pixel intensity distribution along $t$ coordinate is presented in Fig. 1(d). We reconstruct a thickness map of the object for 50 wavelengths and the results are presented as HS cube $64 \times 64 \times 50$. The accuracy of the reconstruction is characterized by the relative-root-mean-square error (RRMSE) criterion calculated for each wavelength:

$$
\operatorname{RRMSE}=\frac{\sqrt{\left\|\hat{h}_{\text {est }}-h_{\text {true }}\right\|_{2}^{2}}}{\sqrt{\left\|h_{\text {true }}\right\|_{2}^{2}}},
$$

where $\hat{h}_{\text {est }}$ and $h_{\text {true }}$ are the reconstructed and true thickness maps, respectively. RRMSE values $<0.1$ correspond to visually high-quality $2 \mathrm{D}$ imaging.

We perform experiments with an additive Gaussian noise of different $\sigma$ to test the algorithm's robustness to noise. The noise level with respect to the spectral signals at the sensor plane is characterized by the peak-signal-to-noise ratio (PSNR) calculated as

$$
\operatorname{PSNR}=10 \log _{10}\left[\frac{\max _{x, y, k}\left(\left|B_{k}\right|\right)}{\sigma}\right]
$$

where $\max _{x, y, k}\left(\left|B_{k}\right|\right)$ is the maximum of the intensity spectra at the sensor plane.

The estimates of the object thickness obtained from the phases of the entire HS cube according to Eq. (18) are presented in Fig. 2 as 2D and 3D images. A nearly perfect correspondence of these estimates to the true thickness images in Fig. 1 is clear. In these tests: iteration number

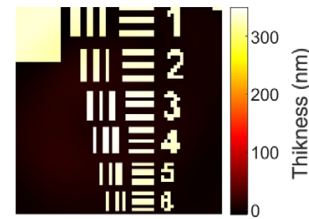

(a)

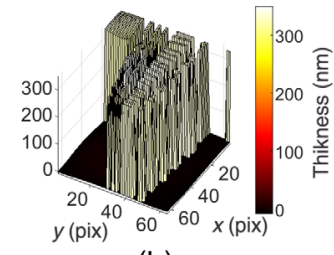

(b)

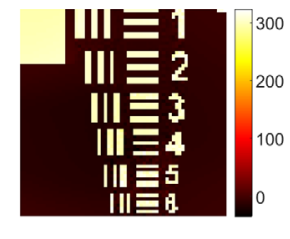

(c)

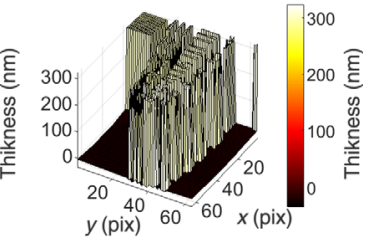

(d)

Fig. 2 Reconstruction of the object thickness obtained by the HS phase retrieval, iteration number $s=30$, (a), (b) HSPR, PSNR $=29.8 \mathrm{~dB}$, RRMSE $=0.031, \sigma=0$ and (c), (d) HSPR, $\mathrm{PSNR}=12.2 \mathrm{~dB}$, RRMSE $=0.11, \sigma=15$. 


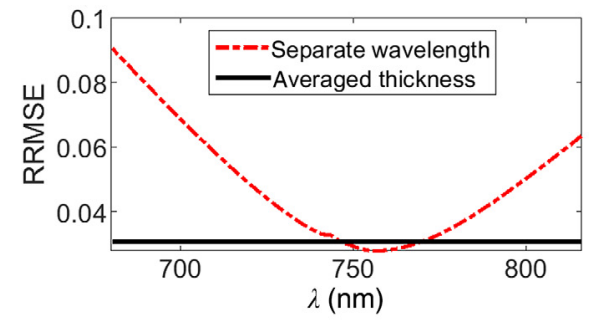

(a)

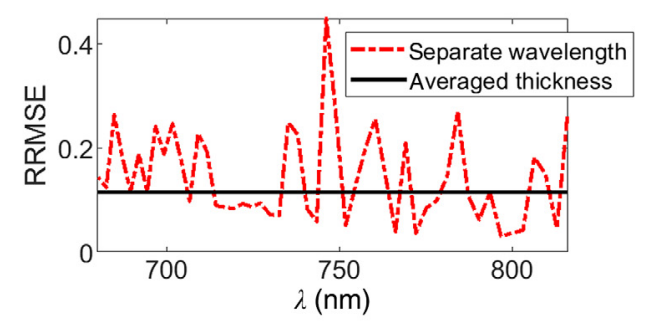

(b)

Fig. 3 RRMSE curves for reconstructed thicknesses from observations: (a) $\sigma=0$ (PSNR = $29.8 \mathrm{~dB}$ ) and $(\mathrm{b}) \sigma=15(\mathrm{PSNR}=12.2 \mathrm{~dB}$ ). Dash red curves are for reconstructed objects' thickness from separate wavelengths and solid black curves are for reconstructed objects' thickness averaged along the whole range of wavelengths.

$s=30, \sigma=0$, and $\sigma=15$. The low values of RRMSEs prove the high-accuracy performance of the algorithm achieved for a small number of iterations.

Let us compare two ways of thickness reconstruction: from the phase estimate obtained for a single wavelength and from the reconstructed entire complex-valued cube where the data for all wavelengths are used simultaneously. In the first case, the thickness is calculated by Eq. (16) and, in the second case, according to Eq. (18).

A red dashed curve in Fig. 3(a), the low-noise case (PSNR $=29.8 \mathrm{~dB}$ ) is obtained for separate estimates of thickness from single-wavelength phase estimates. The minimum value of RRMSE is achieved near the middle point of the wavelength interval. The solid-black curve shows RRMSE achieved by the estimate obtained for thickness of the entire reconstructed phase cube. Naturally, this RRMSE value is invariant on wavelength. Note that this RRMSE value is quite near to the smallest value achieved by the red-dashed curve.

The curves in Fig. 3(b) are obtained for much noisier observations (PSNR $=12.2 \mathrm{~dB}$ ). The reconstruction by Eq. (18) (solid-black line) is wavelength invariant and shows higher RRMSE as compared with the corresponding curve in Fig. 3(a). A red-dashed curve is varying with a lot of up and down peaks. This behavior of the estimates differs from what we had in Fig. 3(a). We may conclude that for the noisier data, the separate thickness estimates are very noisy and unstable with wavelength. At the same time, the estimate based on the joint use of all wavelength estimates gives the results that can be treated as much better than those for most wavelengths. As we do not know in advance the best wavelength for thickness estimation, the estimate [Eq. (18)] guarantees the high accuracy for thickness reconstruction for both noisy and noiseless cases.

The fast convergence rate of the algorithm is demonstrated in Fig. 4 showing RRMSE as a function of iteration number $s$ for different noise levels and different wavelengths. Figure 4(a)

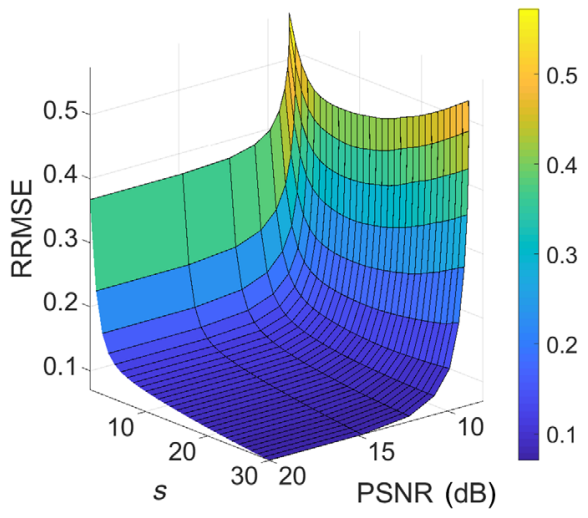

(a)

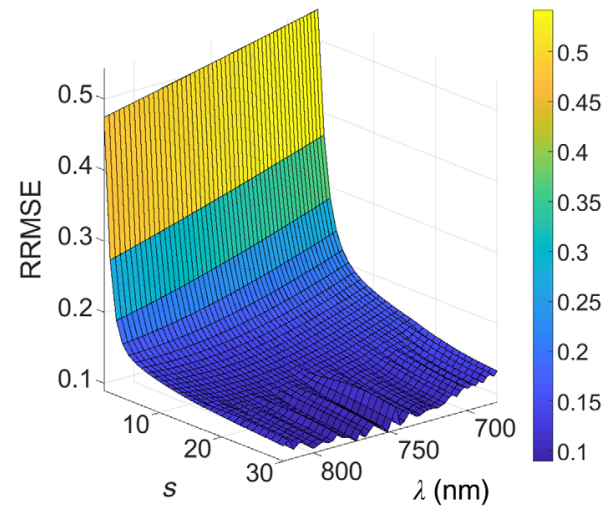

(b)

Fig. 4 RRMSE maps for the reconstructions in different noise conditions. (a) Map of RRMSE values averaged over $\lambda$ as function of iteration number $s$ and PSNR and (b) RRMSE for each $\lambda$ depending on iteration number $s$ for the case of PSNR $=12.2 \mathrm{~dB}$. 
presents RRMSE values averaged over $\lambda$ for the entire HS cube depending on the given noise level, measured by PSNR, Eq. (20). Figure 4(b) shows the RRMSE values for PSNR = $12.2 \mathrm{~dB}$ and for each wavelength. These images prove that the algorithm is robust to noise and provides eligible low RRMSE values even for very noisy observations of PSNR $=12.2 \mathrm{~dB}$. Due to the spectral uniformity of the modeled illumination source and the joint HS cube processing with CCF, RRMSEs for different wavelengths achieve similar values. For both RRMSE maps, the dark blue regions correspond to high-quality reconstructions.

\subsection{Physical Tests}

For experimental validation, we consider a biological specimen, a fly wing that has both amplitude and phase-varying features. The experimental data were obtained by the lensless optical system implementing the principles of Fourier spectrometry with a supercontinuum laser source $\Lambda=[650: 900] \mathrm{nm}$ and a piezo-driven stage in the delay line. ${ }^{11}$ The optical setup is presented in Fig. 5(a), where the interferometric scheme is realized. Figure 5(b) shows the spectrum of the laser source registered by the commercial spectrometer (Thorlabs CCS200), dashed blue curve, and reconstructed by a Fourier transform, solid orange curve. For the commercial spectrometer curve, we applied the quantum efficiency of the camera. These two curves are in close agreement with each other.

We recorded $l_{K}=1880$ observations $Z_{t}(x, y)$ with the phase-delaying step of $59.7 \mathrm{~nm}$. The total distance of the delay line defines the spectral resolution of the wavenumber as $\Delta k=44.6 \mathrm{~cm}^{-1}$. Due to the non-uniformity of the laser spectrum, the object reconstruction is performed for only 50 wavelengths with relatively high-intensity spectra in the range $\Lambda=[681: 802] \mathrm{nm}$. The results of the amplitude and phase reconstruction for slice $\lambda=736 \mathrm{~nm}$ are shown in Fig. 6. It is clearly seen that both amplitude and phase features are well distinguishable in the provided images. The wrapped phase regions appear in the phase image (sharp black lines), however, they do not corrupt the reconstructions since the algorithm CCF provides filtering for complex-domain wavefronts, where no distinction exists between absolute and wrapped phases. The HS cube of phase/amplitude reconstruction for each slice (wavelength) is presented in Video 1.

For demonstration of the spatial differences in the spectral responses of the specimen, we show phase and amplitude spectra obtained for three different points of the specimen [see Figs. 7(a) and 7(b)]. Each of the spectral curves corresponds to one of the numbered colored points in the amplitude image [Fig. 7(c)] with the same corresponding colors.

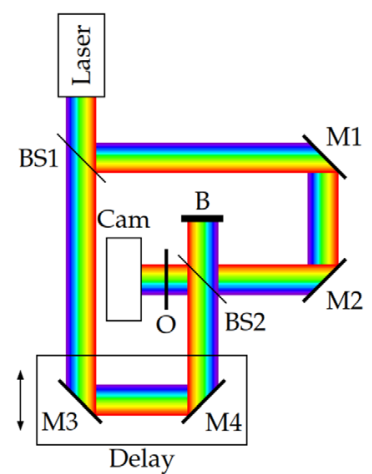

(a)

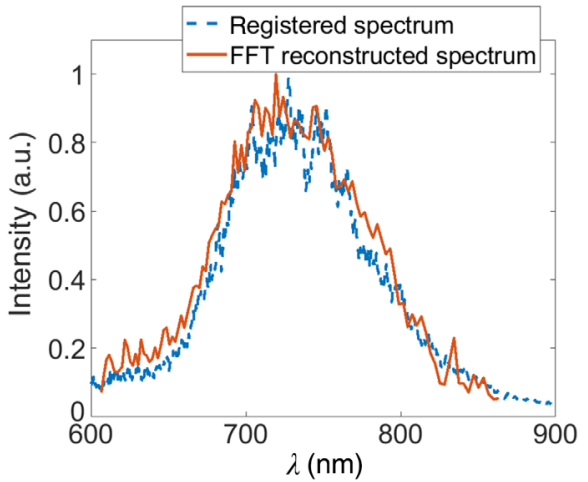

(b)

Fig. 5 (a) HS phase retrieval setup. BS1 and BS2 are beamsplitters, M1 to M4 are mirrors, "O" is a transparent specimen, "Cam" is a registering sensor, " $\mathrm{B}$ " is a light blocker, and "Delay" is a moving delay stage. (b) Used light spectrum: a blue-dashed curve is a spectrum registered by a spectrometer and multiplied by the camera quantum efficiency. An orange solid curve is a spectrum reconstructed by our algorithm. 

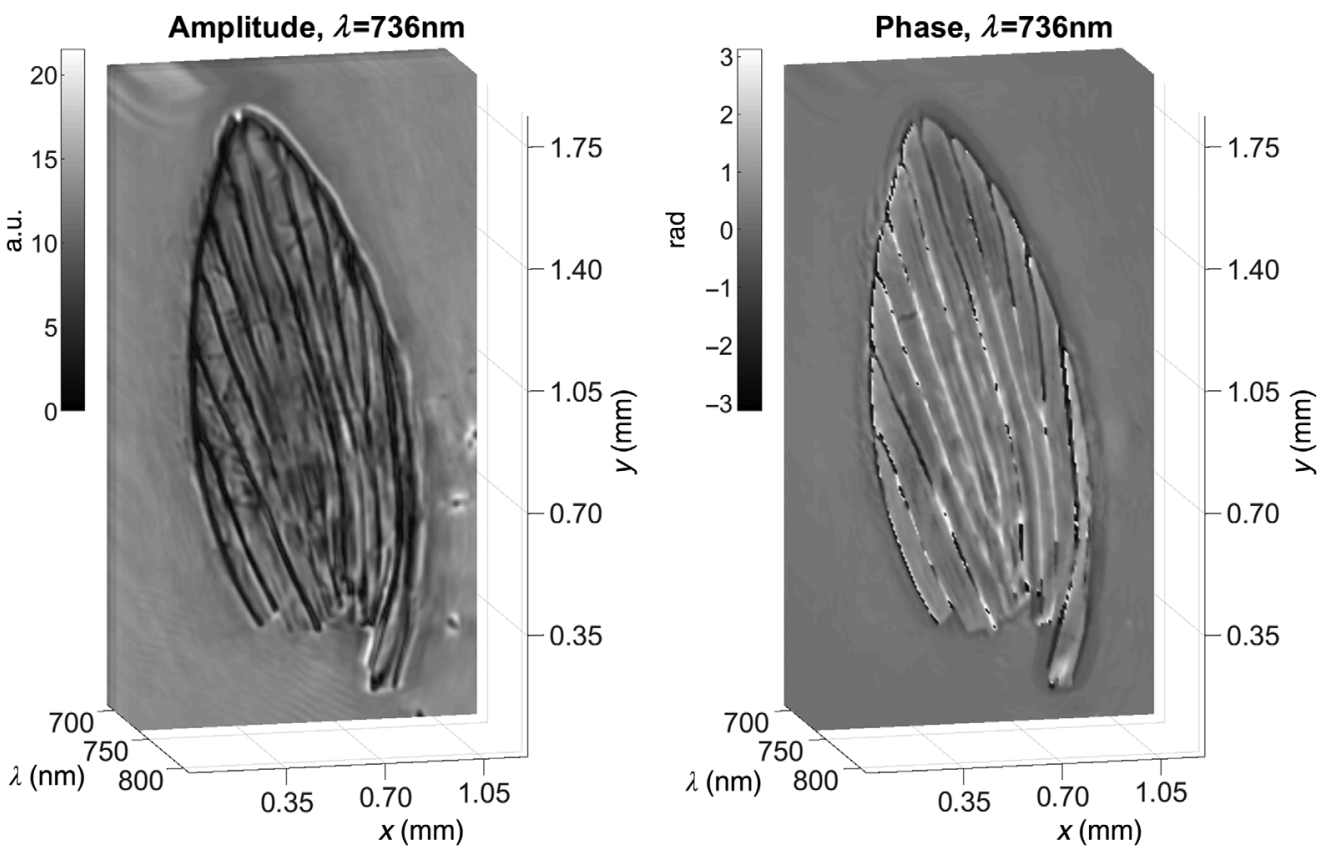

Fig. 6 The amplitude and phase reconstructions corresponding to the $\lambda=736 \mathrm{~nm}$ slice of the 3D cube. The amplitudes and phases for the entire reconstructed HS cube can be seen in Video 1 (Video 1, MP4, 1.193 MB [URL: https://doi.org/10.1117/1.OE.60.1.013108.1]).

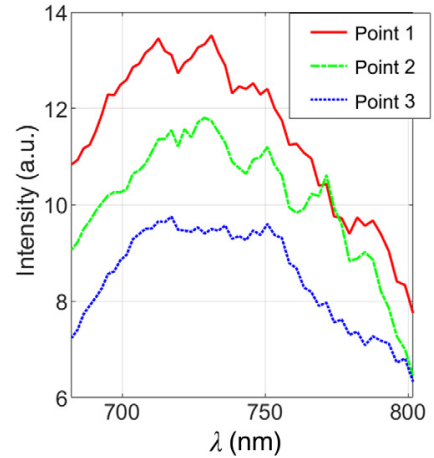

(a)

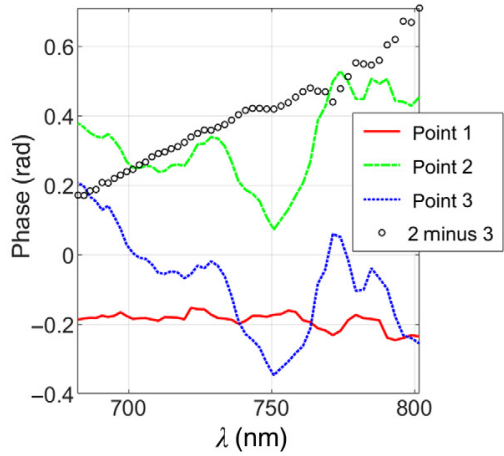

(b)

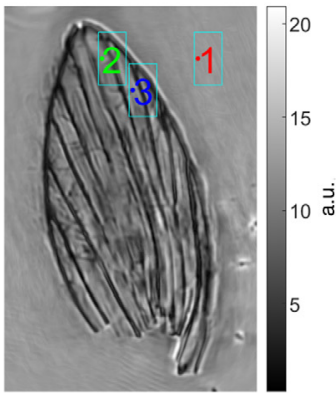

(c)

Fig. 7 (a) Amplitude and (b) phase spectra for different points of the object. The curves in the left and middle plots correspond to the points numbered in (c) the amplitude image, $\lambda=745 \mathrm{~nm}$ with the same colors. Black circles curve in plot (b) is a phase difference between curves from points 2 and 3 .

The point " 1 " is located out of the specimen area and, therefore, the amplitude spectrum in Fig. 7(a) represents the laser spectrum multiplied on the registration camera quantum efficiency. The corresponding phase spectrum curve in Fig. 7(b) is nearly invariant on wavelength.

The points " 2 " and " 3 " belong to the specimen and reflect different spectrum absorbance and disturbances in different areas of the wing. These spatial variations in phase and amplitude cannot be interpreted unambiguously as variations in both thickness and refractive index can cause them.

The black circle curve in Fig. 7(b) is a difference of the phase curves for points " 2 " and " 3 ." It is growing approximately linearly with a growing wavelength. If we assume that the wavelengthdependent refractive index is the same for these two points, such a behavior of this curve indicates that the object in point 2 is thinner than in point 3 . In this way, sometimes the ambiguity of thickness/refractive index can be overcome. 
For the experiments, we used MATLAB R2020a on a computer with 32 GB of RAM and $1.9 \mathrm{GHz}$ Intel ${ }^{\circledR}$ Core $^{\mathrm{TM}}$ i7-08665U CPU. A single iteration for HS cube with dimensions $1500 \times 1500 \times 50$ took around $150 \mathrm{~s}$.

\section{Conclusion}

An HS phase retrieval algorithm has been developed and presented, which allows the reconstruction of the complex amplitude of a transparent specimen in the lensless optical setup based on Fourier spectrometry principles in a shearographic geometry. This self-referencing lensless system enables a large field of view and robustness of the results with respect to vibration. A specimen phase lost in intensity measurements is reconstructed by the developed iterative algorithm utilizing HS complex domain sparsity of wavefronts. The advantage of HS data processing compared to the results available for monochromatic experiments is demonstrated. With the growing interest in HS measurements, we expect that the developed algorithm finds applications in various bio- and medical tasks concerning noninvasive quantitative phase imaging.

\section{Appendix A: Minimization on $B_{\text {I }}$}

For minimization $\min _{\left\{B_{l}\right\}} J$, we calculate the derivative $\partial J / \partial B_{l}^{*}$ and solve the equation $\partial J / \partial B_{l}^{*}=0$ what leads to the complex valued solution of the form $B_{l}=\left|B_{l}\right| e^{j \varphi_{B_{l}}}$.

The following manipulations define the derivatives $\partial J / \partial B_{l}^{*}, l=1,2, \ldots, N / 2-1$ :

$$
\begin{aligned}
\partial J / \partial B_{l}^{*} & =\frac{-2}{\sigma^{2}} \sum_{t=0}^{N-1}\left[\Delta Z_{t}-2 \sum_{k=1}^{N / 2-1}\left|B_{k}\right|^{2} \cos \left(\frac{2 \pi}{N} k t\right)\right]\left[2 \cos \left(\frac{2 \pi}{N} l t\right) B_{l}\right]+\frac{1}{\gamma}\left(B_{l}-A_{l} U_{o, l}\right) \\
& =\frac{-4}{\sigma^{2}} \sum_{t=0}^{N-1} \Delta Z_{t} \cos \left(\frac{2 \pi}{N} l t\right) B_{l}+\frac{8}{\sigma^{2}} \sum_{k=1}^{N / 2-1}\left|B_{k}\right|^{2} \frac{N}{2} \delta_{k, l} B_{l}+\frac{1}{\gamma}\left(B_{l}-A_{l} U_{o, l}\right) \\
& =\frac{-4}{\sigma^{2}} \sum_{t=0}^{N-1} \Delta Z_{t} \cos \left(\frac{2 \pi}{N} l t\right) B_{l}+\frac{4 N}{\sigma^{2}}\left|B_{l}\right|^{2} B_{l}+\frac{1}{\gamma}\left(B_{l}-A_{l} U_{o, l}\right) .
\end{aligned}
$$

It is used in the second line of these equations that

$$
\sum_{t=0}^{N-1} \cos \left(\frac{2 \pi}{N} k t\right) \cos \left(\frac{2 \pi}{N} l t\right)=\frac{N}{2} \delta_{k, l}, \quad \text { for } k, l=1, \ldots, N / 2-1,
$$

where $\delta_{k, l}$ is the Kronecker's symbol, $\delta_{k, l}=1$ for $k=l$ and otherwise $\delta_{k, l}=0$.

It follows from the third line in Eq. (22) that the complex-valued $B_{l}$ is a solution of Eq. (8).

\section{Acknowledgments}

This research was supported by Jane and Aatos Erkko Foundation, Finland, and Finland Centennial Foundation: Computational Imaging without Lens (CIWIL) project. The authors declare no conflict of interest. The founding sponsors had no role in the design of the study; in the collection, analysis, or interpretation of data; in the writing of the manuscript, and in the decision to publish the results.

\section{References}

1. C. Dorrer et al., "Spectral resolution and sampling issues in Fourier-transform spectral interferometry," J. Opt. Soc. Am. B 17(10), 1795-1802 (2000).

2. S. Kalenkov, G. Kalenkov, and A. Shtanko, "Spectrally-spatial Fourier-holography," Opt. Express 21(21), 24985-24990 (2013). 
3. V. T. Tenner, K. S. Eikema, and S. Witte, "Fourier transform holography with extended references using a coherent ultra-broadband light source," Opt. Express 22(21), 2539725409 (2014).

4. S. Eisebitt et al., "Lensless imaging of magnetic nanostructures by x-ray spectro-holography," Nature 432(7019), 885 (2004).

5. D. N. Naik et al., "Spectrally resolved incoherent holography: 3D spatial and spectral imaging using a Mach-Zehnder radial-shearing interferometer," Opt. Lett. 39, 1857-1860 (2014).

6. D. Claus et al., "Accuracy enhanced and synthetic wavelength adjustable optical metrology via spectrally resolved digital holography," J. Opt. Soc. Am. A: Opt. Image Sci. Vision 35(4), 546-552 (2018).

7. Á. B. Peña et al., "Refractive index properties of the retina accessed by multi-wavelength digital holographic microscopy," Proc. SPIE 10883, 108830X (2019).

8. Y. Hung, "Shearography: a new optical method for strain measurement and nondestructive testing," Opt. Eng. 21(3), 213391 (1982).

9. R. J. Bell, Introductory Fourier Transform Spectroscopy, Academic Press (1972).

10. S. G. Kalenkov, G. S. Kalenkov, and A. E. Shtanko, "Self-reference hyperspectral holographic microscopy," J. Opt. Soc. Am. A 36, A34 (2019).

11. I. Shevkunov, V. Katkovnik, and K. Egiazarian, "Lensless hyperspectral phase imaging in a self-reference setup based on Fourier transform spectroscopy and noise suppression," Opt. Express 28(12), 17944 (2020).

12. P. Bao et al., "Lensless phase microscopy using phase retrieval with multiple illumination wavelengths," Appl. Opt. 51(22), 5486-5494 (2012).

13. V. Y. Katkovnik, I. A. Shevkunov, and K. Eguiazarian, "Hyperspectral phase retrieval," Proc. SPIE 11351, 113511G (2020).

14. B. Roig-Solvas, L. Makowski, and D. H. Brooks, "A proximal operator for multispectral phase retrieval problems," SIAM J. Optim. 29(4), 2594-2607 (2019).

15. R. Bell, Introductory Fourier Transform Spectroscopy, Elsevier (2012).

16. V. Katkovnik, I. Shevkunov, and K. Egiazarian, "Hyperspectral holography and spectroscopy: computational features of inverse discrete cosine transform," arXiv:1910.03013 (2019).

17. F. Soulez et al., "Proximity operators for phase retrieval," Appl. Opt. 55(26), 7412-7421 (2016).

18. N. Parikh et al., "Proximal algorithms," Found. Trends® Optim. 1(3), 127-239 (2014).

19. J. W. Goodman, Introduction to Fourier Optics, Roberts and Company Publishers (2005).

20. S. V. Venkatakrishnan, C. A. Bouman, and B. Wohlberg, "Plug-and-play priors for model based reconstruction," in IEEE Global Conf. Signal Inf. Process., IEEE, pp. 945-948 (2013).

21. C. A. Metzler, A. Maleki, and R. G. Baraniuk, "Bm3d-prgamp: compressive phase retrieval based on bm3d denoising," in IEEE Int. Conf. Image Process. (ICIP), IEEE, pp. 2504-2508 (2016).

22. V. Katkovnik, M. Ponomarenko, and K. Egiazarian, "Sparse approximations in complex domain based on BM3D modeling," Signal Process. 141, 96-108 (2017).

23. V. Katkovnik and K. Egiazarian, "Sparse phase imaging based on complex domain nonlocal BM3D techniques," Digital Signal Process.: Rev. J. 63, 72-85 (2017).

24. J. M. Bioucas-Dias and J. M. Nascimento, "Hyperspectral subspace identification," IEEE Trans. Geosci. Remote Sens. 46(8), 2435-2445 (2008).

25. L. Zhuang and J. M. Bioucas-Dias, "Fast hyperspectral image denoising and inpainting based on low-rank and sparse representations," IEEE J. Sel. Top. Appl. Earth Obs. Remote Sens. 11, 730-742 (2018).

26. V. Katkovnik et al., "Complex-domain joint broadband hyperspectral image denoising," Sens. Transducers 233(5), 33-39 (2019).

27. I. Shevkunov et al., "Hyperspectral phase imaging based on denoising in complex-valued eigensubspace," Opt. Lasers Eng. 127, 105973 (2020).

28. J. Bioucas-Dias and G. Valadão, "Multifrequency absolute phase estimation via graph cuts," in Eur. Signal Process. Conf., pp. 1389-1393 (2009). 
29. Y. Park, C. Depeursinge, and G. Popescu, "Quantitative phase imaging in biomedicine," Nat. Photonics 12(10), 578-589 (2018).

30. M. Born and E. Wolf, Principles of Optics: Electromagnetic Theory of Propagation, Interference and Diffraction of Light, Elsevier (2013).

31. P. Hartmann, Optical Glass, SPIE Press, Bellingham, Washington (2014).

Vladimir Katkovnik received his $\mathrm{PhD}$ and $\mathrm{DSc}$ degrees in technical cybernetics from Leningrad Polytechnic Institute (LPI) in 1964 and 1974, respectively. From 1964 to 1991, he was an associate professor and then a professor in the Department of Mechanics and Control Processes at LPI. Since 2003, he has been in the Department of Signal Processing, Tampere University of Technology, Finland. He published 6 books and more than 350 refereed journal and conference papers. His research interests include stochastic image/signal processing, nonparametric estimation, computational imaging, and computational phase imaging.

Igor Shevkunov received his $\mathrm{PhD}$ in optics from St. Petersburg State University, Saint Petersburg, Russia, in 2013. He has been a postdoctoral researcher at Tampere University since 2017. He is the author of more than 45 refereed papers. His main research interests are digital holography, phase retrieval, and interferometry.

Karen Egiazarian received his MSc degree from Yerevan State University in 1981, his PhD from Moscow State University, Moscow, Russia, in 1986, and his DTech degree from Tampere University of Technology (TUT), Tampere, Finland, in 1994. He is a professor leading the Computational Imaging Group of the ICT Faculty at Tampere University. He has authored about 650 refereed journal and conference papers. His research interests include computational imaging, sparse coding, and image and video restoration. He serves as an associate editor for the IEEE Transactions on Image Processing and was the editor-in-chief of the Journal of Electronic Imaging from 2016-2020. 